\title{
Analysing the Capability of the Catchment's Spectral Signature for the Regionalization of Hydrological Parameters
}

\author{
Laura Fragoso-Campón ${ }^{1}$, Pablo Durán-Barroso ${ }^{1}$, and Elia Rosado² \\ ${ }^{1}$ Universidad de Extremadura \\ ${ }^{2}$ Universidad de Extremadura Escuela Politecnica
}

May 14, 2021

\begin{abstract}
Water resource management in ungauged catchments is complex due to the uncertainties around the hydrological parameters that dominate the streamflow behaviour. These parameters are usually defined by regionalization approaches in which hydrological response patterns are transferred from gauged to ungauged basins. Regression-based methods using physical properties derived from cartographic data sources are widely used. The current remote sensing techniques offer us new standpoints in regionalisation processing since the hydrological response depends on the physical attributes related to the spectral responses of the territory. Moreover, machine learning approaches have not been specifically applied to the regionalization of hydrologic parameters. This work studies the capability of a catchment's spectral response based on Sentinel-1 and Sentinel-2 data to address a regression-based regionalization of hydrological parameters using a machine learning approach. Hydrological modelling was conducted by the HBV-light model. We tested the random forest algorithm in several regionalization scenarios: the new approach using the catchments' spectral signature, the traditional method using physical properties and a fusion of them. The calibration results were excellent (median KGE $=0.83$ ), and the regionalized parameters obtained with the random forest algorithm achieved good performance in which the three scenarios showed almost the same goodness of fit (median KGE $=$ 0.45 to 0.50$)$. We found that the effectiveness depends on the climatic environment and that predictions in humid catchments exhibited better performance than those in the driest catchments. The physical approach (median KGE $=0.71$ ) exhibited better performance than the spectral approach (median KGE $=0.64$ ) in humid catchments, whereas spectral regionalization (median $\mathrm{KGE}=0.33$ ) outperformed the physical scenario in the driest catchments (median KGE $=0.25$ ). Herein, our results confirm that regionalization is still challenging in Mediterranean climate variants where the new spectral approach showed promising results and time series of satellite data could improve seasonal regionalization methodologies.
\end{abstract}

\section{Hosted file}

Manuscript_Fragoso_et_al_main_text.pdf available at https://authorea.com/users/413858/ articles/522036-analysing-the-capability-of-the-catchment-s-spectral-signature-forthe-regionalization-of-hydrological-parameters 

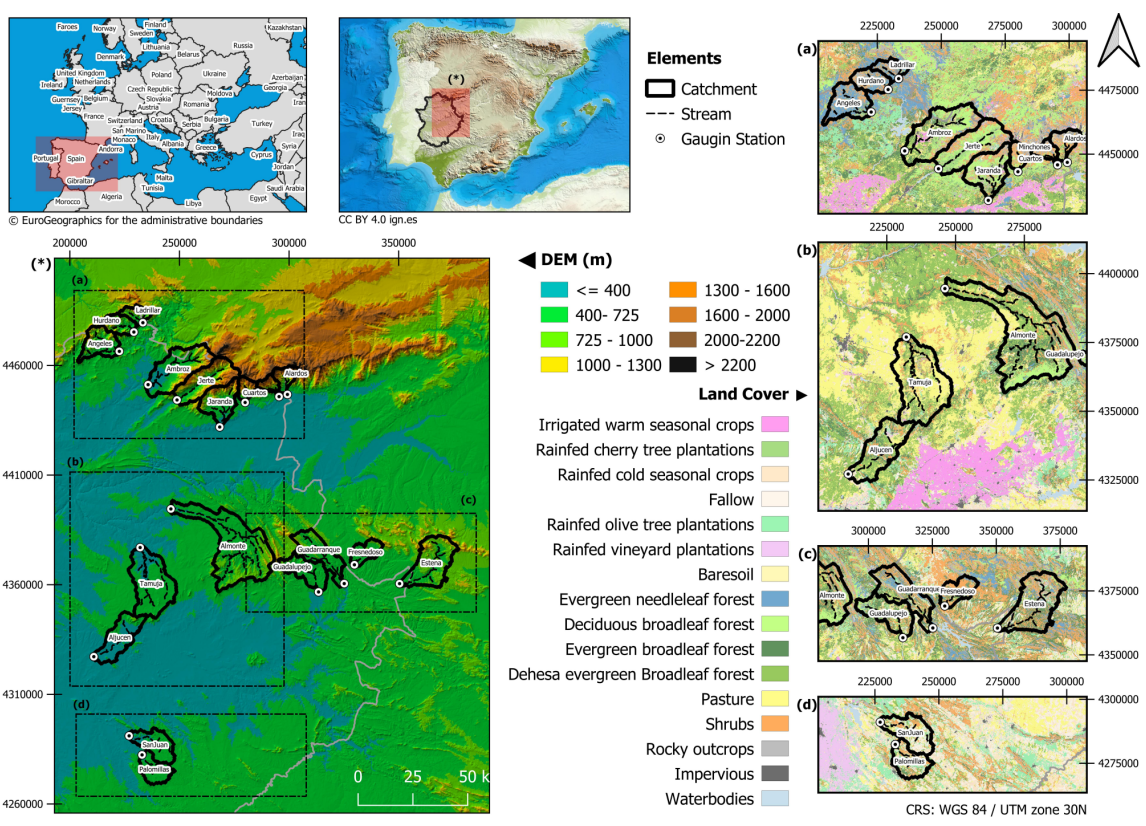

$<=400 \square 1300-1600$ 400-725 1600-2000

$725-1000 \square 2000-2200$

$1000-1300$

$>2200$

Irrigated warm seasonal crops

Rainfed cherry tree plantations

Rainfed cold seasonal crops

Fallow
Rainfed olive tree plantations Rainfed vineyard plantations

Baresoil

Evergreen needleleaf forest Deciduous broadleaf forest

Evergreen broadleaf forest

Dehesa evergreen Broadleaf forest

$$
\begin{array}{r}
\text { Pasture } \\
\text { Shrubs } \\
\text { Rocky outcrops } \\
\text { Impervious }
\end{array}
$$
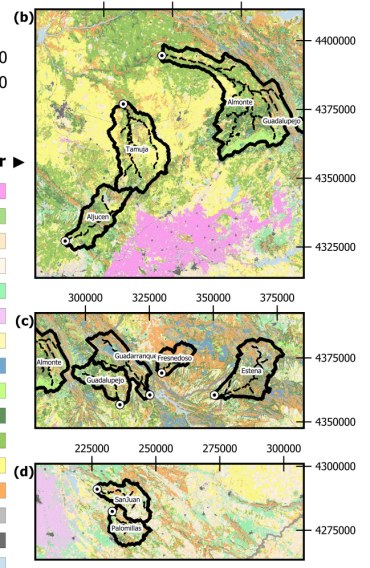

CRS: WGS 84 / UTM ZOne 30N

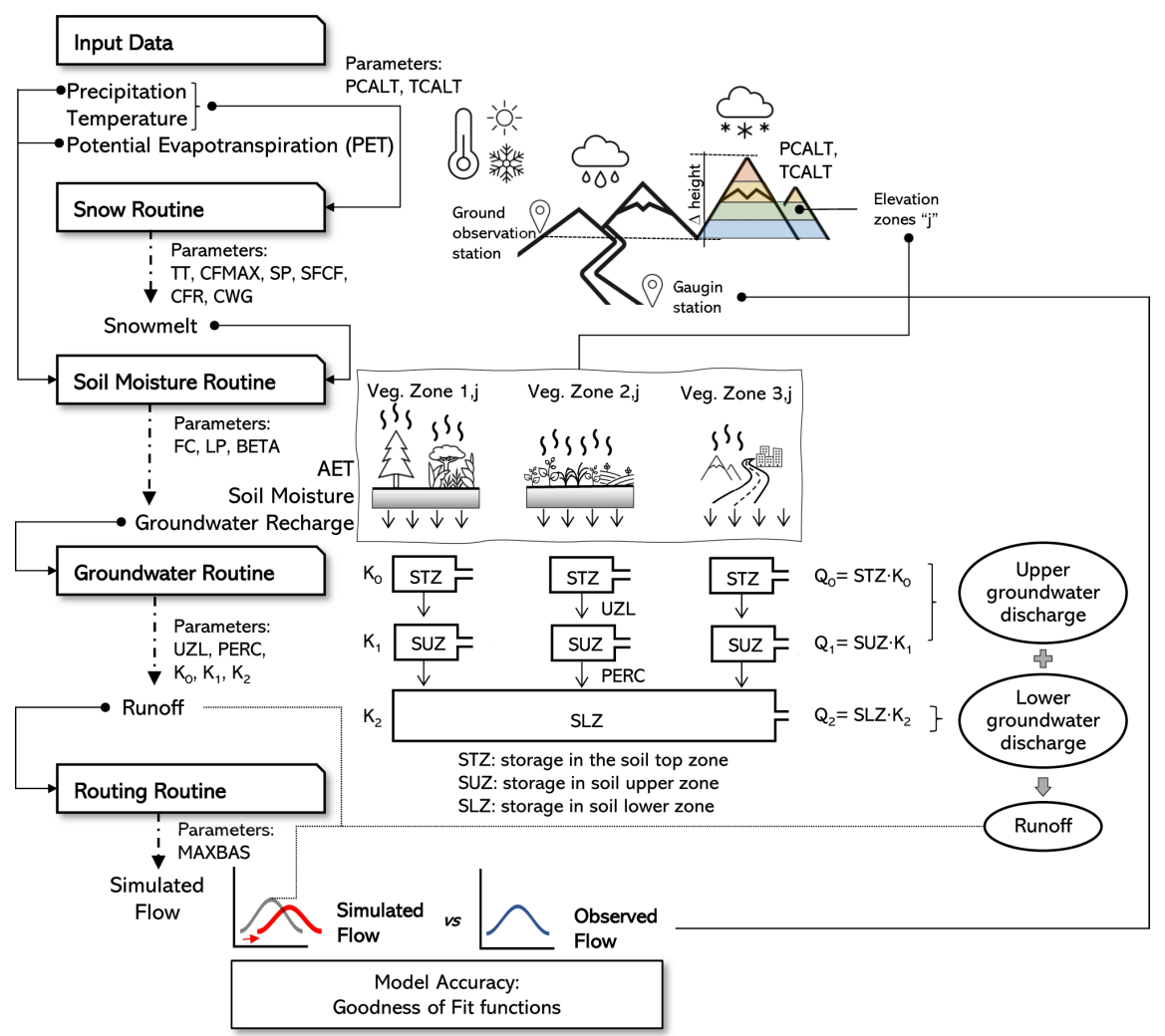




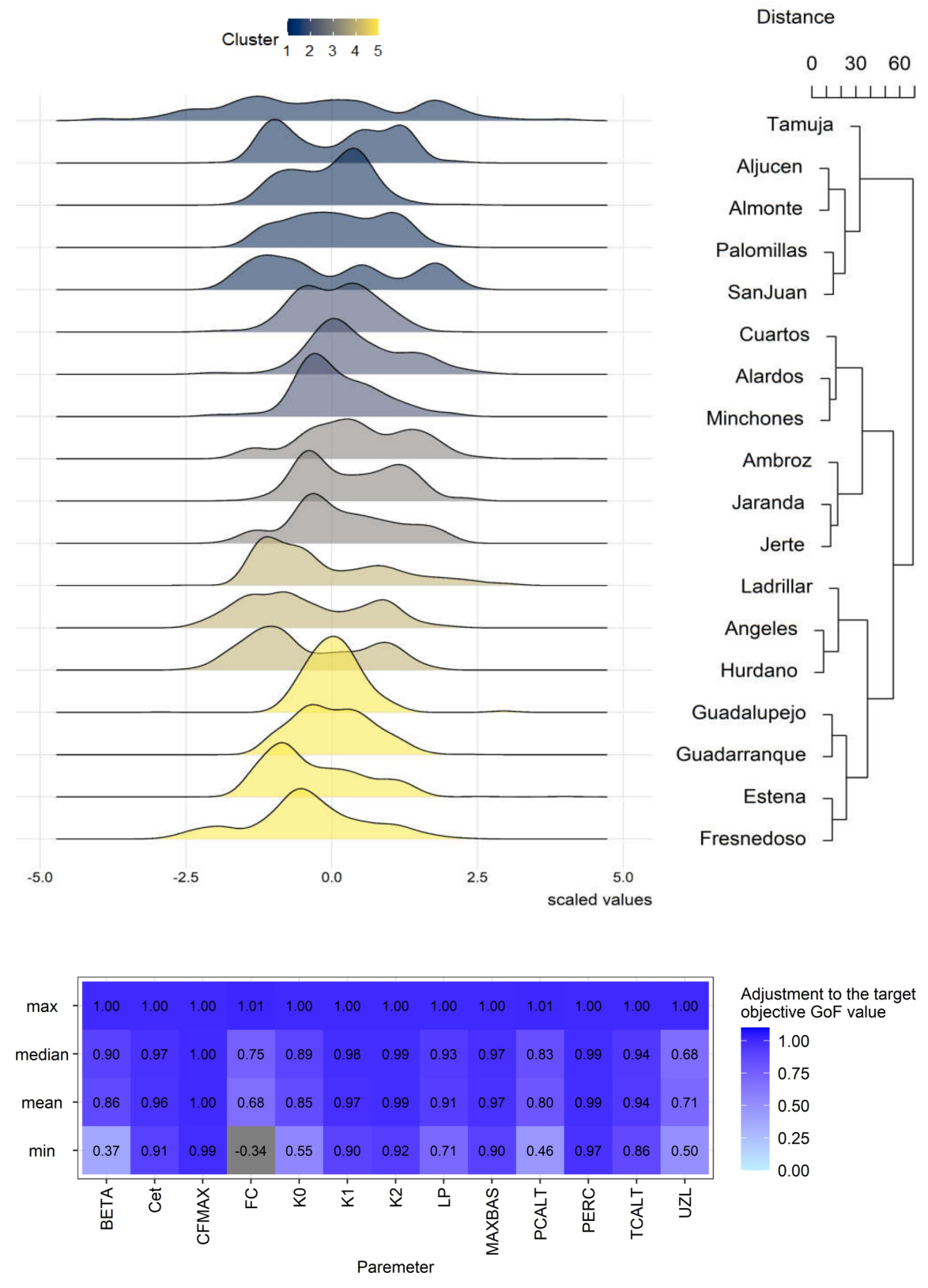



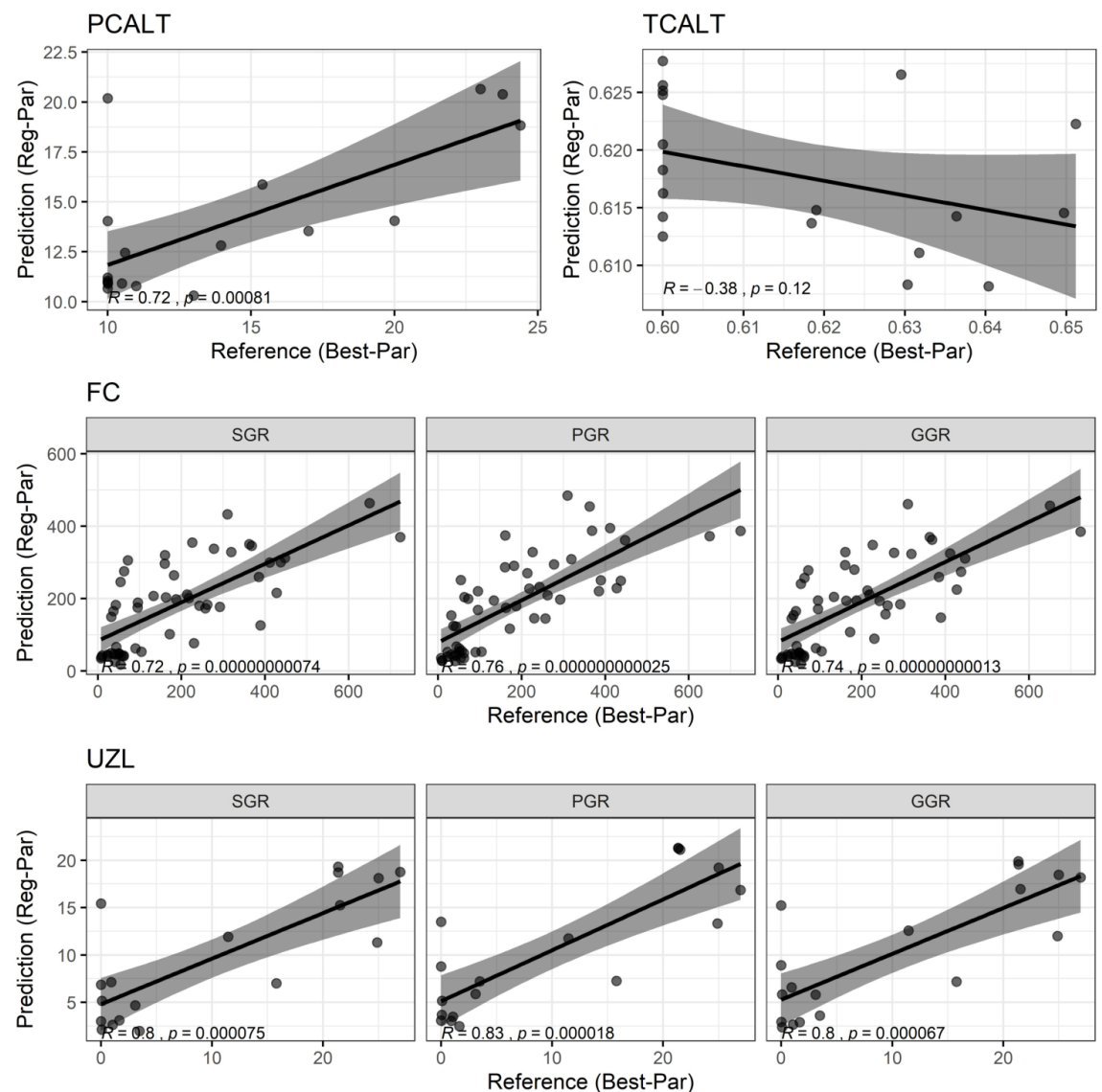

KO
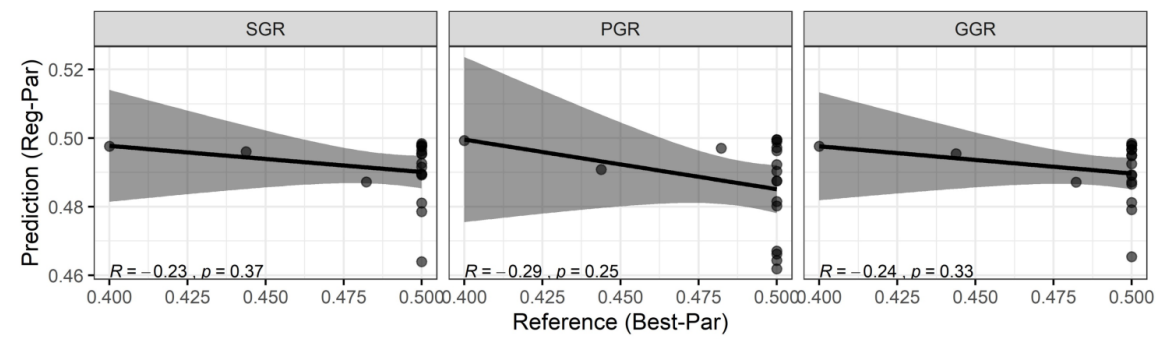


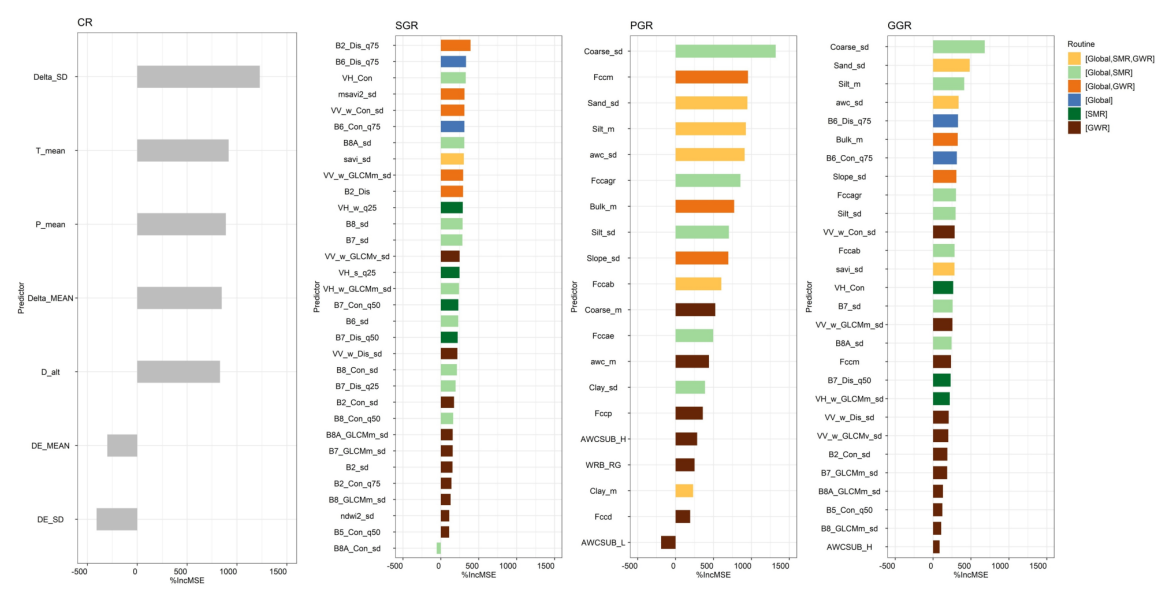



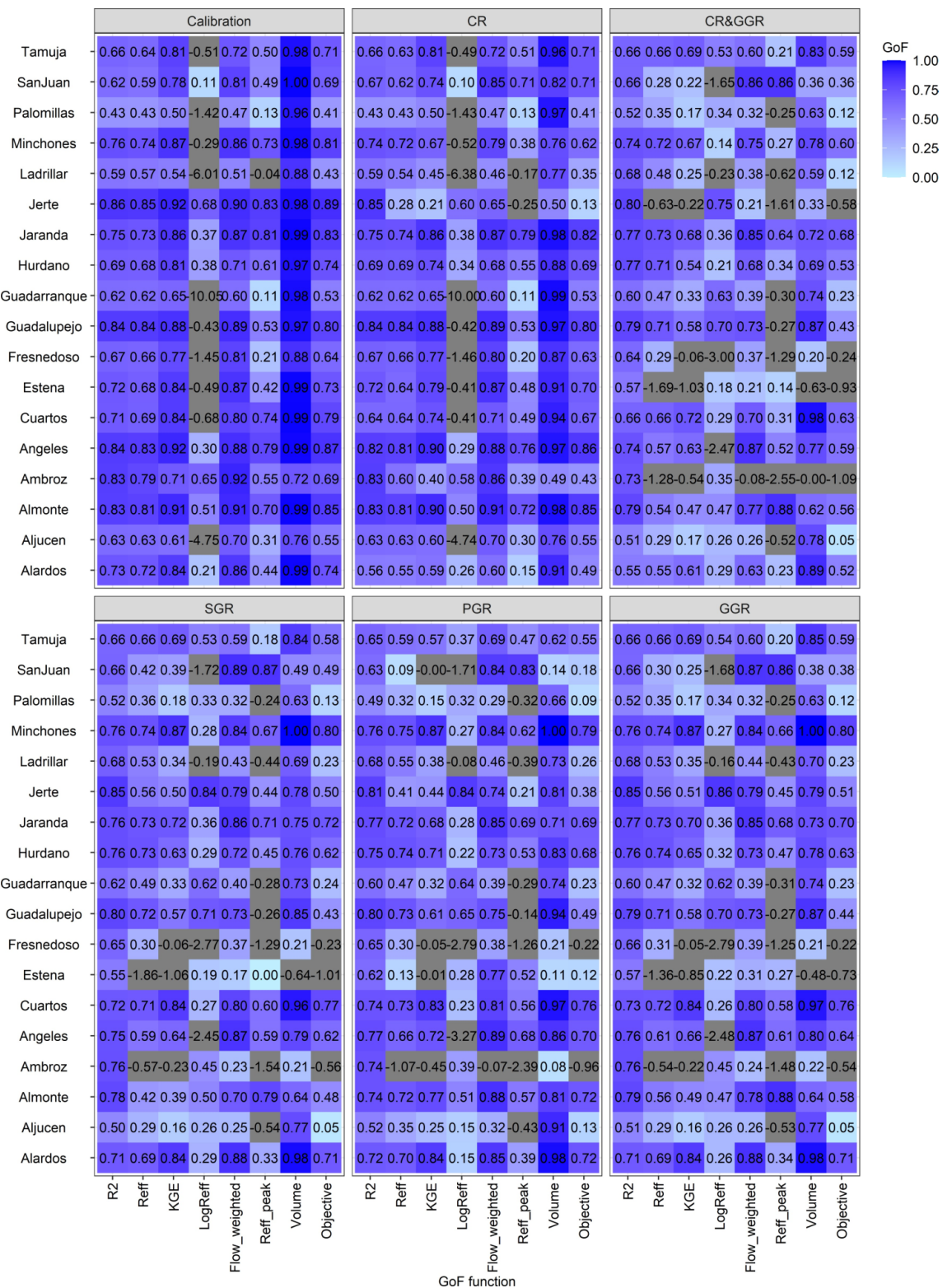

$0.80-0.63-0.220 .750 .21-1.610 .33-0.58$

0.770 .730 .680 .360 .850 .640 .720 .68 0.770 .710 .540 .210 .680 .340 .690 .53 $0.600 .470 .330 .630 .39-0.300 .740 .23$ $0.790 .710 .580 .700 .73-0.270 .870 .43$ $0.640 .29-0.06-3.000 .37-1.290 .20-0.24$ $0.57-1.69-1.030 .180 .210 .14-0.63-0.93$ 0.660 .660 .720 .290 .700 .310 .980 .63 $0.740 .570 .63-2.470 .870 .520 .770 .59$ $0.73-1.28-0.540 .35-0.08-2.55-0.00-1.09$ 0.790 .540 .470 .470 .770 .880 .620 .56 $0.510 .290 .170 .260 .26-0.520 .780 .05$ 0.550 .550 .610 .290 .630 .230 .890 .52

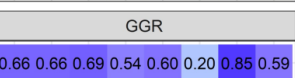
$0.660 .300 .25-1.680 .870 .860 .380 .38$ $0.520 .350 .170 .340 .32-0.250 .630 .12$ 0.760 .740 .870 .270 .840 .661 .000 .80 $0.680 .530 .35-0.160 .44-0.430 .700 .23$ 0.850 .560 .510 .860 .790 .450 .790 .51 0.770 .730 .700 .360 .850 .680 .730 .70 0.760 .740 .650 .320 .730 .470 .780 .63 $0.600 .470 .320 .620 .39-0.310 .740 .23$ $0.790 .710 .580 .700 .73-0.270 .870 .44$ $0.660 .31-0.05-2.790 .39-1.250 .21-0.22$ $0.57-1.36-0.850 .220 .310 .27-0.48-0.73$ 0.730 .720 .840 .260 .800 .580 .970 .76 $0.760 .610 .66-2.480 .870 .610 .800 .64$ $0.76-0.54-0.220 .450 .24-1.480 .22-0.54$ 0.790 .560 .490 .470 .780 .880 .640 .58 $0.510 .290 .160 .260 .26-0.530 .770 .05$ 0.710 .690 .840 .260 .880 .340 .980 .71

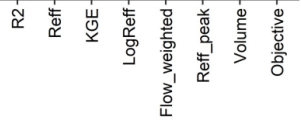



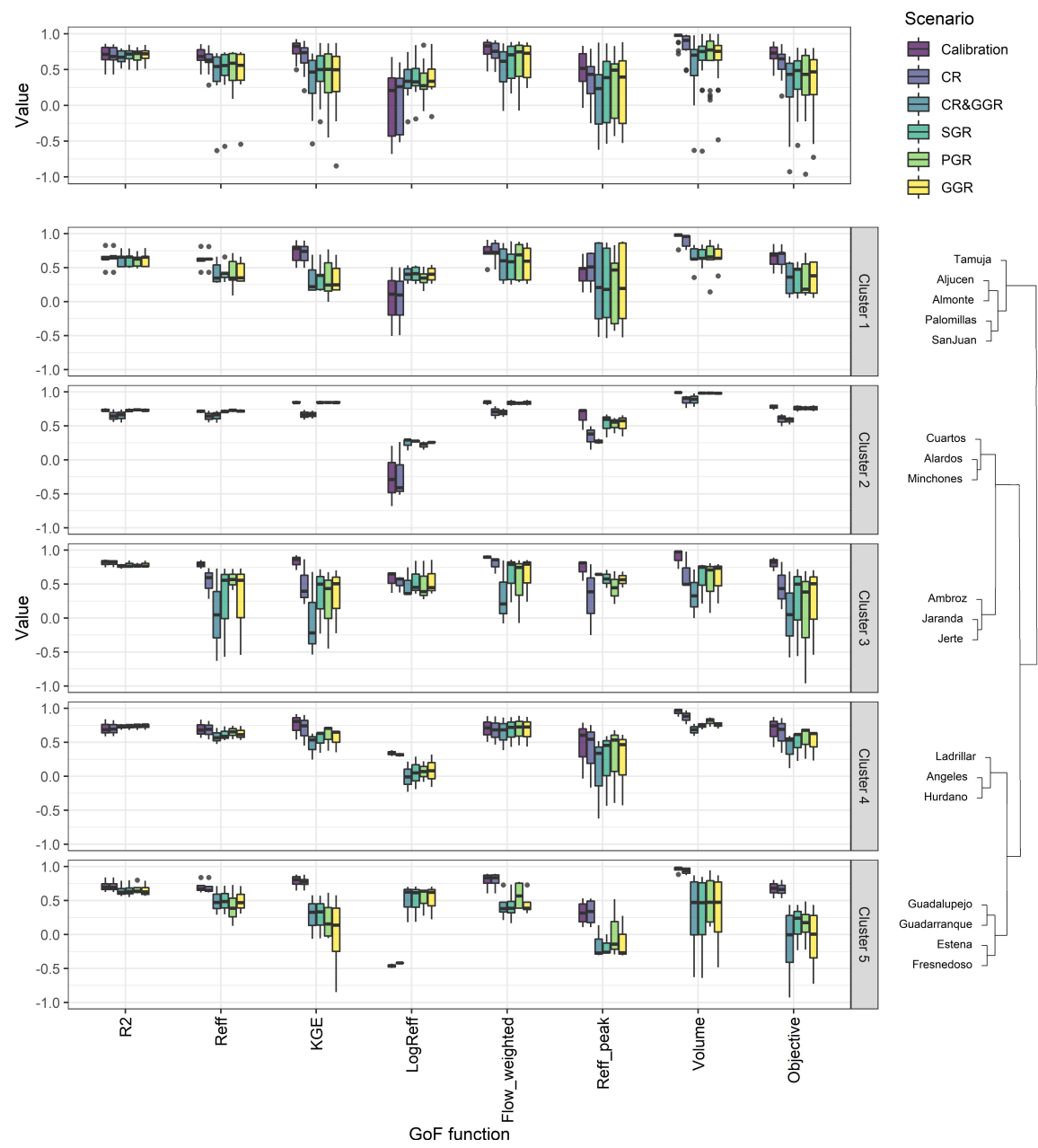
Catchment: Angeles
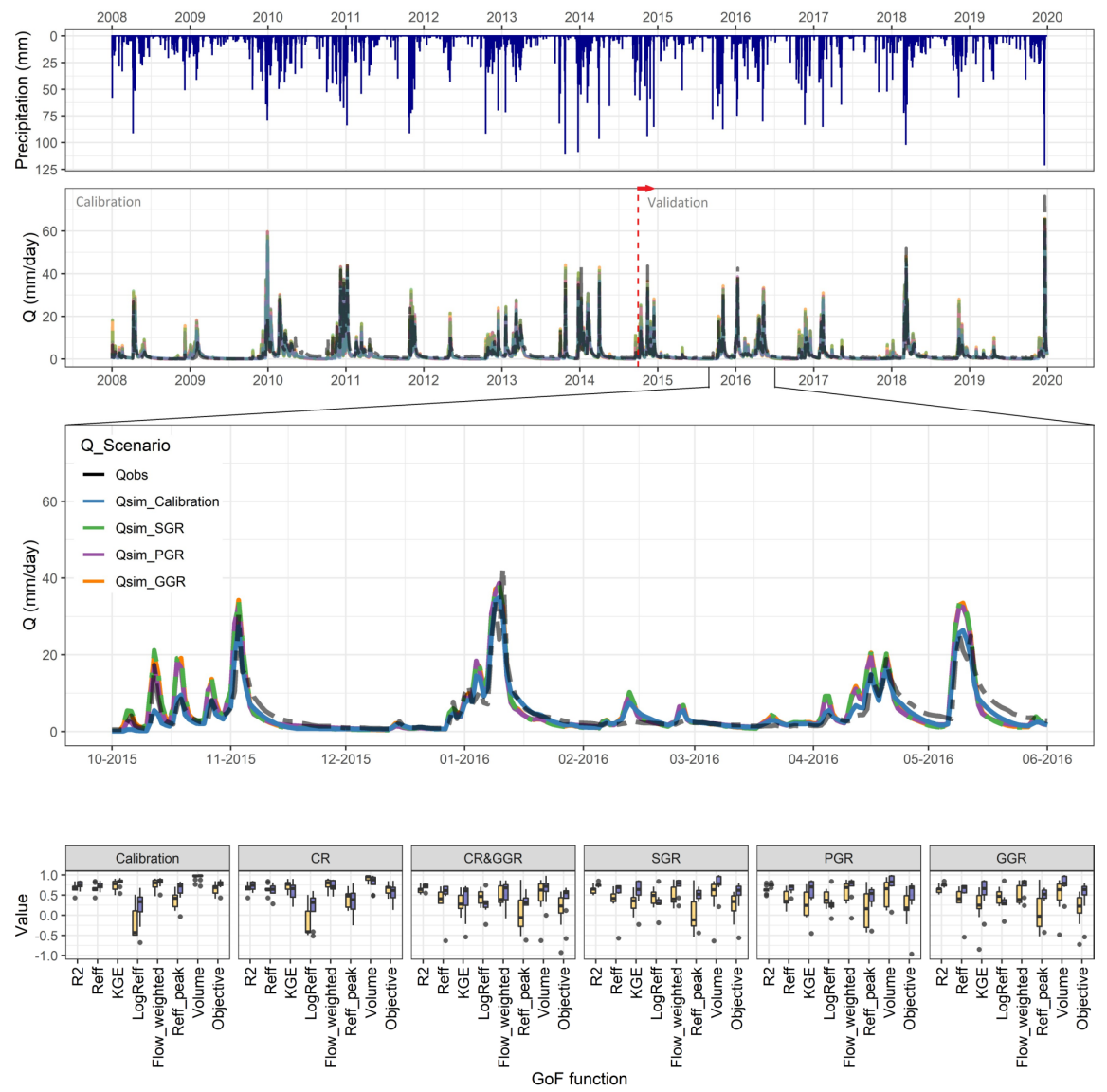

Bioclimatic variant 官 Mpc 官 Tocsm
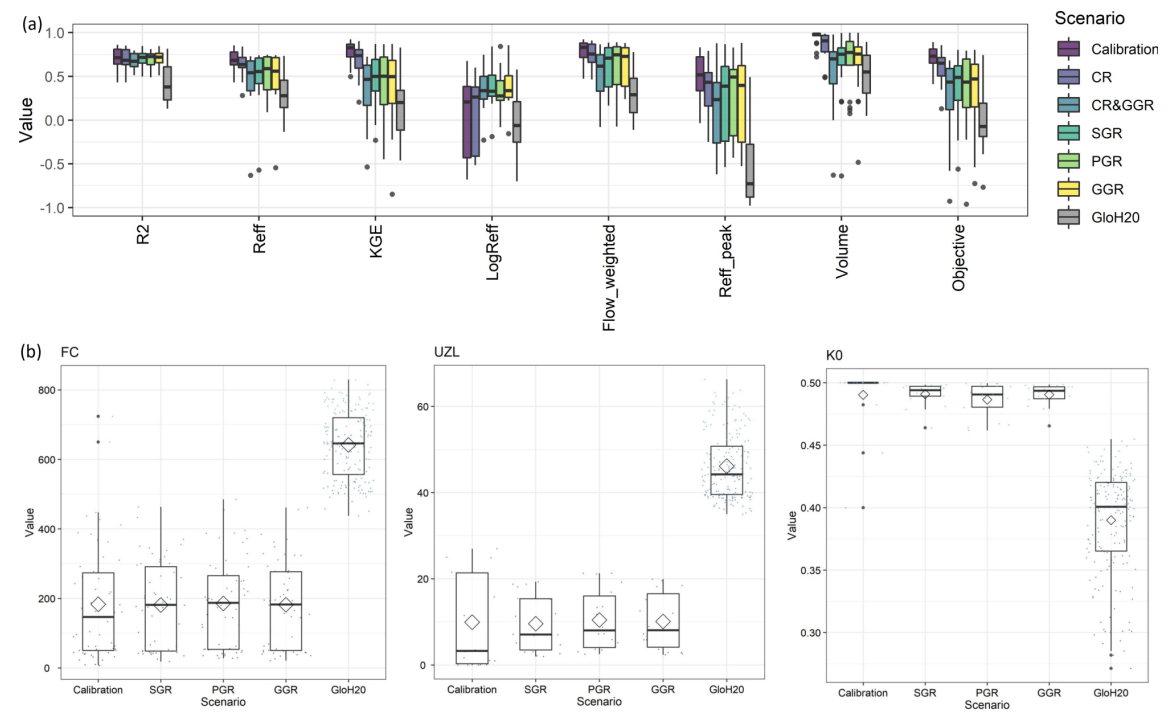

Hosted file 
Table_01.pdf available at https://authorea.com/users/413858/articles/522036-analysingthe-capability-of-the-catchment-s-spectral-signature-for-the-regionalization-ofhydrological-parameters

\section{Hosted file}

Table_02.pdf available at https://authorea.com/users/413858/articles/522036-analysingthe-capability-of-the-catchment-s-spectral-signature-for-the-regionalization-ofhydrological-parameters

\section{Hosted file}

Table_03.pdf available at https://authorea.com/users/413858/articles/522036-analysingthe-capability-of-the-catchment-s-spectral-signature-for-the-regionalization-ofhydrological-parameters

\section{Hosted file}

Table_04.pdf available at https://authorea.com/users/413858/articles/522036-analysingthe-capability-of-the-catchment-s-spectral-signature-for-the-regionalization-ofhydrological-parameters

\section{Hosted file}

Table_05.pdf available at https://authorea.com/users/413858/articles/522036-analysingthe-capability-of-the-catchment-s-spectral-signature-for-the-regionalization-ofhydrological-parameters

\section{Hosted file}

Table_06.pdf available at https://authorea.com/users/413858/articles/522036-analysingthe-capability-of-the-catchment-s-spectral-signature-for-the-regionalization-ofhydrological-parameters

\section{Hosted file}

Table_07.pdf available at https://authorea.com/users/413858/articles/522036-analysingthe-capability-of-the-catchment-s-spectral-signature-for-the-regionalization-ofhydrological-parameters

\section{Hosted file}

Table_08.pdf available at https://authorea.com/users/413858/articles/522036-analysingthe-capability-of-the-catchment-s-spectral-signature-for-the-regionalization-ofhydrological-parameters 